\title{
Come predire le fratture da fragilità oltre i dieci anni? Lo studio OFELY
}

\author{
Fabio Lanfranco $\cdot$ Stefano Allasia
}

Pubblicato online: 17 gennaio 2015

(C) Springer International Publishing AG 2015

\section{Commento a:}

How to predict fragility fracture beyond ten years?

The OFELY study.

\section{E. Sornay-Rendu, F. Duboeuf, S. Boutroy,}

R.D. Chapurlat.

J Clin Endocrinol Metab (2014) 99(12):4690-4697

L'osteoporosi e il conseguente aumento del rischio di frattura rappresenta uno dei principali problemi di salute per le donne in post-menopausa. La maggior parte degli studi presenti in letteratura ha avuto una durata inferiore ai dieci anni, pertanto il dato della densità minerale ossea (Bone Mineral Density, BMD) come unico elemento nel predire a lungo termine il rischio di fratture è scarsamente suffragato da dati scientifici. Lo studio OFELY ha coinvolto, tra il 1992 e il 1993, 867 donne francesi di età $\geq 40$ anni (media $59 \pm 10$ anni), di cui 680 in post-menopausa e 187 in pre-menopausa, seguite per un tempo medio di 20 anni. Gli autori hanno valutato il rischio della prima frattura da fragilità considerando i valori basali di BMD ottenuti mediante dual-energy X-ray absorptiometry (DXA), l'indice FRAX (rischio fratturativo entro dieci anni) e le condizioni cliniche evidenziate durante la prima visita, in grado di alterare la normale omeostasi scheletrica.

In 20 anni di follow-up 245 donne hanno manifestato una o più fratture da fragilità, tutte documentate all'RX, 64 a livello vertebrale e 181 in sede non vertebrale. Le donne che sono andate incontro a prima frattura dopo 10 anni dall'inizio dello studio (Fx $10-20, \mathrm{~N}=109)$ erano più

F. Lanfranco ( $\varangle) \cdot$ S. Allasia

Divisione di Endocrinologia, Diabetologia e Metabolismo,

Dipartimento di Scienze Mediche, Università di Torino, Torino,

Italia

e-mail: fabio.lanfranco@unito.it giovani e presentavano un più basso indice FRAX rispetto alle donne con frattura nei primi 10 anni di osservazione (Fx 0-10, N = 136). Dopo correzione dei dati per l'età, il gruppo Fx 10-20 rispetto al gruppo Fx 0-10 presentava ancora valori basali significativamente più alti di BMD sul sito lombare e una maggiore forza di presa misurata con il dinamometro. La diminuzione di una DS della BMD misurata a livello dei corpi vertebrali, collo del femore, femore totale e radio distale si correlava a un aumentato rischio di frattura oltre i 10 anni, rispettivamente di 1,43 (IC 95\%, 1,12-1,82), 1,39 (IC 95\%, 1,08-1,82), 1,47 (IC 95\%, 1,14-1,89) e 2,0 volte (IC 95\%, 1,47-2,7). Infine, nelle donne con diagnosi densitometrica di osteoporosi il rischio fratturativo a 10 anni e oltre i 10 anni aumentava in modo significativo rispetto alle donne con normale BMD, mentre il quadro di osteopenia non si associava a un maggiore rischio di fratture nel tempo.

In conclusione, questo studio prospettico ha evidenziato che una ridotta BMD e una storia familiare di fratture di femore rappresentano i principali fattori di rischio per fratture da fragilità. Nel tempo i fattori di rischio clinici assumono, invece, minore importanza nel predire le fratture da fragilità, soprattutto nella popolazione anziana di età superiore ai 70 anni. Lo studio sottolinea che la BMD rappresenta il determinante principale per stimare il rischio di frattura da fragilità sia a breve che a lungo termine in donne di età superiore ai 40 anni. La presenza di valori alterati di BMD può essere utilizzata nella pratica clinica per prendere in considerazione l'inizio di terapie farmacologiche tempestive e promuovere stili di vita in grado di prevenire l'insorgenza di fratture osteoporotiche nel tempo. 